\title{
IEA PVPS Task 16 - Solar resource for high penetration and large scale applications
}

\section{Summary}

The joint Task of IEA Technological Collaboration Programmes PVPS and SolarPACES started in July 2017 and will last till 2020. The Task 16 is also collaborating with SHC. 21 countries and 57 institutions are currently part of the Task. It offers a unique platform of exchange for private and public organizations as well as data providers and science organizations. A strong emphasis on extra gain of collaboration will be promoted. Benchmarks, standardizations, state-of the art descriptions and dissemination will be in the focus. Main added values are: lower resource uncertainties and higher forecast accuracies, which will lead to lower implementation costs of PV and CSP projects.

At SWC we will show the state of the work. The second year was used to exercise benchmarks and workshops. Three benchmarks were organized. One about methods to calculate regional power forecasts, a second about forecasts based on all sky imagers and a third one about site adaptation methods. Three workshops where held. The first workshop covered best practices for automatic and expert based quality control procedures and gap filling methods, the second probabilistic forecasting and the third - during SWC 19 - benchmarking of site adaptation methods.

solar energy, solar resources, forecasts, global radiation, collaboration

\section{State of the Task}

A short introduction of the Task 16 (http://www.iea-pvps.org/index.php?id=389) organization, institutions, workplan (Table 1) and objectives will be given. The focus of the paper is the work done in the 2nd year of the Task. This includes mainly the three workshops and the three benchmarking exercises.

Table 1: Scope of the three Subtasks

\begin{tabular}{|l|l|}
\hline Subtask & Scope \\
\hline $\begin{array}{l}\text { Subtask 1: Evaluation of } \\
\text { current and emerging resource } \\
\text { assessment methodologies }\end{array}$ & $\begin{array}{l}\text { This subtask is focusing on the evaluation of current and emerging resource } \\
\text { assessment methodologies. Different methodologies are analysed and } \\
\text { conclusions are formulated in the form of best practices guidelines and/or } \\
\text { standards. } \\
\text { The three methods (ground based methods, Numerical Weather Prediction } \\
\text { models (NWP) and satellite-based methods - are evaluated in this subtask. For } \\
\text { each methodology a separate activity is defined. }\end{array}$ \\
\hline $\begin{array}{l}\text { Subtask 2: Enhanced data \& } \\
\text { bankable products }\end{array}$ & $\begin{array}{l}\text { Subtask 2 is mainly dedicated to end-users, notably in the PV domain. It is } \\
\text { focusing on the main PV applications of the different types of solar resource } \\
\text { products and datasets. End-users needs in concentrating solar thermal, solar } \\
\text { heating and buildings will also be considered. }\end{array}$ \\
\hline $\begin{array}{l}\text { Subtask 3: Evaluation of } \\
\text { current and emerging solar } \\
\text { resource and forecasting } \\
\text { techniques }\end{array}$ & $\begin{array}{l}\text { Subtask 3 focusses on different aspects of forecast evaluation and comparison. In } \\
\text { particular we will address the economic value of solar forecasting for a variety of } \\
\text { different applications, the topic of regional forecasting important for transmission } \\
\text { operators and variability and probabilistic forecasting. } \\
\text { Depending of the application and the corresponding forecast horizon different } \\
\text { models and input data are applied for solar irradiance and power forecasting. } \\
\text { These include numerical weather predictions for several days ahead, satellite } \\
\text { based cloud motion forecasts for several hours ahead, and sky imager forecasts } \\
\text { for high resolution intra-hour forecasting as well as statistical models for } \\
\text { measurement based forecasting and post-processing of physical model forecasts. } \\
\text { Each of the subtask 3 activities includes all of these different forecasting } \\
\text { approaches. }\end{array}$ \\
\hline
\end{tabular}




\section{Benchmarks}

\subsection{Benchmark of site adaption methods}

The benchmark has been organized by Jesus Polo (Ciemat, ESP). The goal is to progress on correction techniques for long-term datasets, applicable to both PV and CSP.

Site adaptation is referring to the process of improving or correcting long term solar radiation modelled time series by comparing to short term ground measurements. Bias removal and correction of systematic errors in the modelled solar radiation data can be performed by using different statistical methods to the deviations found between overlapped ground and modelled data. The methodologies can be divided into two general groups: regression techniques and distribution fitting techniques. Some methodologies have been borrowed from other fields in meteorology or energy meteorology, like wind characterisation or precipitation for instance. In the regression methods the procedures are based on fitting, linear and non-linearly, the errors or deviations between ground and modelled data in such a way that the new regression can be applied to the whole dataset. In the methods based on fitting the distribution, the data is transformed into the distribution domain computing the quantiles and the correction is applied to the quantiles by using the probability distribution function of the ground data.

In the framework of the Task 16 IEA PVPS a benchmarking exercise is being performed where different experts applied different techniques to 10 pairs of datasets (modelled and ground) corresponding to 10 different sites. Modelled data include satellite derived and reanalysis estimations with mostly open and free datasets. Linear regressions and different versions of quantiles mapping techniques are being tested so far.

First results of this benchmark will be shown at the workshop during the SWC 2019 conference.

\subsection{Benchmark of aggregation methods to calculate regional power production forecasting}

The benchmark has been organized by Alessendro Betty (i-em, ITA). Aggregated production for the Italy and Sweden will be used as targets. Three main approaches for regional power production forecasting are possible:

- $\quad$ Bottom-up approach: make the prediction of power output for each PV site of the regional area under consideration and finally aggregate up to the prediction for the whole area (computational demanding, generally not applicable due to missing info)

- Up-scaling method: sample the most representative PV site of each area and forecast PV power production at PV site (or however at a lower level then regional, e.g. cluster of PV sites). Finally upscale power forecast up to regional level (e.g. model outputs average)

- $\quad \mathrm{N}-1$ / Input-Output method: aggregate input meteo data at a lower level than regional and forecast directly power production at regional level (e.g. model inputs average)

The report about this benchmark will be published till the end of 2019.

\subsection{Benchmark of solar power forecast methods based on the use of all sky imagers}

The benchmark organized by Andreas Kazantzidis, GRC and Stefan Wilbert (DLR; DEU), is held at Plataforma Solar de Almeria during summer and autumn 2019.

Several solutions are currently developed to forecast solar power at very short-term (less than $30 \mathrm{~min}$ ). As cloud cover behavior is considered as fully stochastic at this time scale, weather modelling is unable to provide satisfactory forecast. Forecast methods based on real-time observation of weather parameters driving solar energy production are necessary. Among the existing solutions at reasonable cost, the hemispherical vision of the sky with a camera has the advantage to observe the cloud cover surrounding the sun (Figure 1). Therefore, the variation of surface solar irradiance can be anticipated by a prediction based on an observation, not only through statistical modelling completing irradiance measurements. First, clouds are detected in the all sky images. Using two or more cameras, infrared images or other additional sensors such as ceilometers the cloud height can be determined. If the cloud height is known maps of the irradiance on the ground can be calculated. Image series are evaluated to obtain the cloud velocity, which is required to predict the cloud position in the next approximately $15 \mathrm{~min}$. 
State-of-the art of surface solar irradiance forecasting using all-sky camera is relatively recent. However, several experimental and operational solutions emerged since the years 2010 with different approaches in terms of instrument type and forecast algorithms. These approaches show differences in terms of cost, accuracy related to the target application and operational implementation. Today, user requirements are relatively new and users might not be able to qualify the right solution according to their needs. Furthermore, the accuracy of the different all sky imager methods is difficult to compare based on the existing studies: The weather conditions during the different system evaluations vary strongly and the system accuracy strongly depends on these conditions. Therefore a comparative study of such very short-term forecast at the same sit and under the same conditions is relevant. The objectives of the benchmark are the following:

- To qualify the current short-term forecasting solutions based on the use of all-sky cameras

- To provide to users a guidance of existing solutions and their accuracy

- To generate high quality observations on a single site for researchers

- To identify strength or weakness of each approaches

The benchmark will consist in a 3-month observation campaign which will take place at the CIEMAT experimental site in Almeria (Spain): the "Plataforma Solar de Almeria". This campaign is scheduled from August to November 2019. Among these 4 months, at least 30 days with full exploitable data of all instruments are needed. It is required that the data of all instruments are available on these 30 days. The all sky imager forecasts will be compared to more than 10 pyrheliometers and 20 pyranometers operated at PSA by CIEMAT and DLR.

Most of the cameras have already been set up at PSA (Figure 2). The participants of the benchmark including the evaluation team are Uni Patras, CIEMAT, EPFL, Reuniwatt, University of Utrecht, Steady sun, CNRS PROMES / SARL PROMECA, University of Applied Sciences Upper Austria, Meteotest / CSEM, EKO and DLR / CSPS. University of Utrecht and DLR participate with systems of 2 all sky imagers placed approximately $1 \mathrm{~km}$ from each other while the other systems work with one camera.

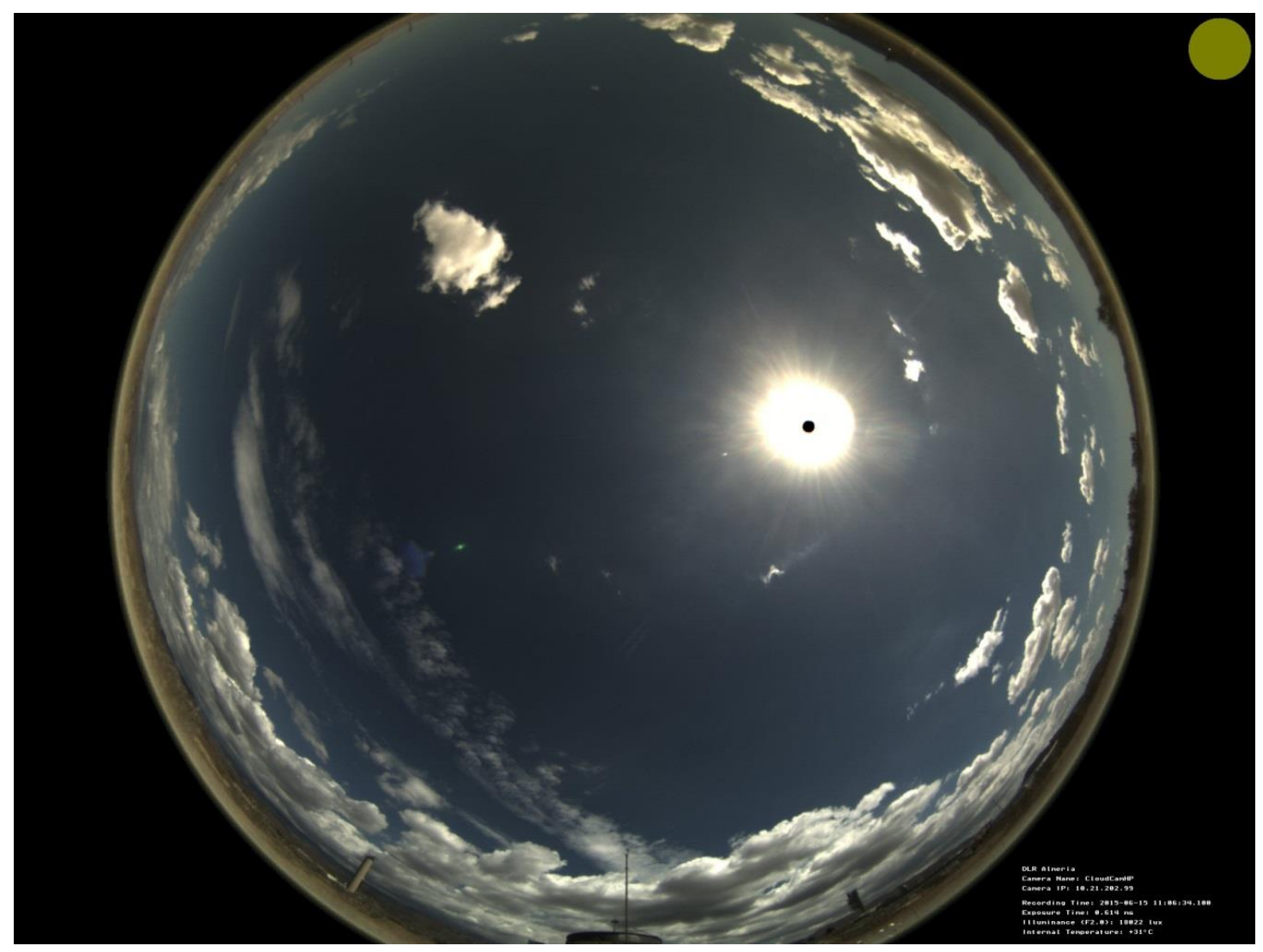

Fig. 1: All sky imager from CIEMAT's Plataforma Solar de Almería. 


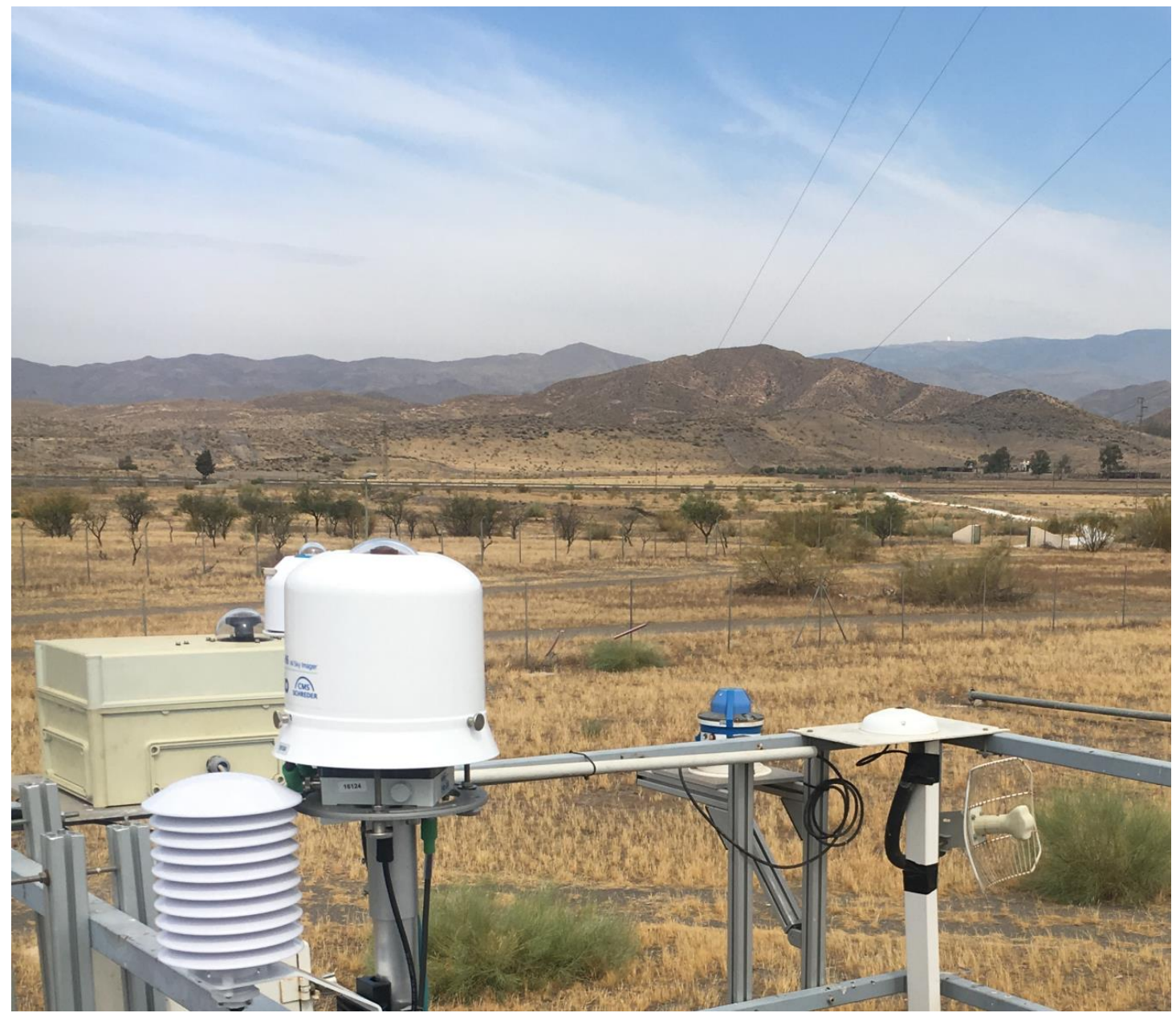

Fig. 1: All sky imagers installed for the system benchmark at CIEMAT's Plataforma Solar de Almería.

\section{Workshops}

3.1. Workshop on best practices for automatic and expert based quality control procedures and gap filling method

This workshop was organized by Philippe Blanc, Mines Paristech as a side event of the ICEM 2019 in Copenhagen, Denmark, June 25th 2019 (http://www.wemcouncil.org/wp/icem2019/). Four presentations were given. Philippe Blanc introduced the general topic and explained why checks are needed. He identified three classes of checks: quality checks based on physical possible limits, consistency checks (based on two or three components) and visual inspections. Especially if DNI is measured visual tests are very useful (Fig. 3 and 4). 


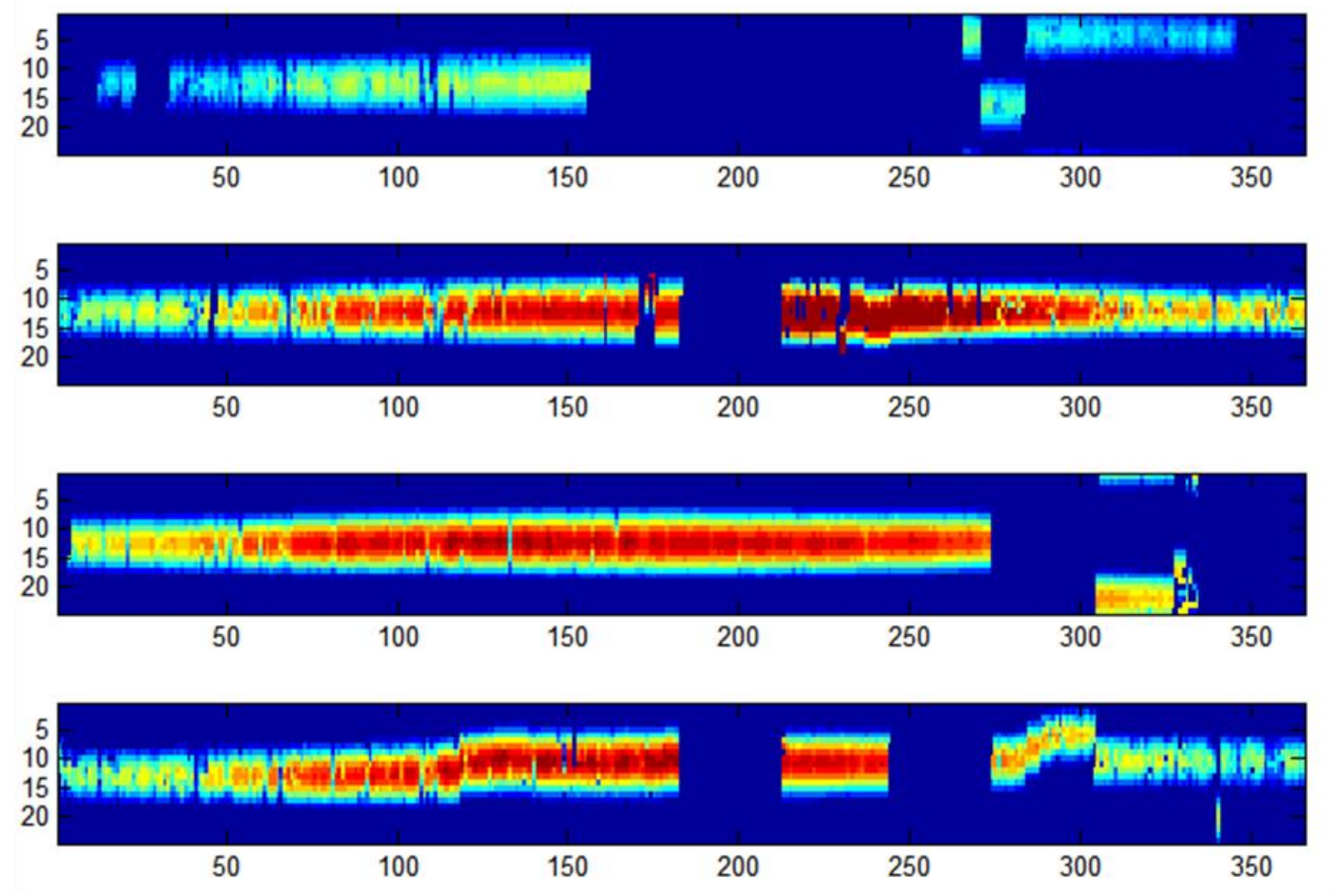

Fig. 3: 2D view of a global radiation timeseries (on year). Missing periods and time shifts are clearly visible.

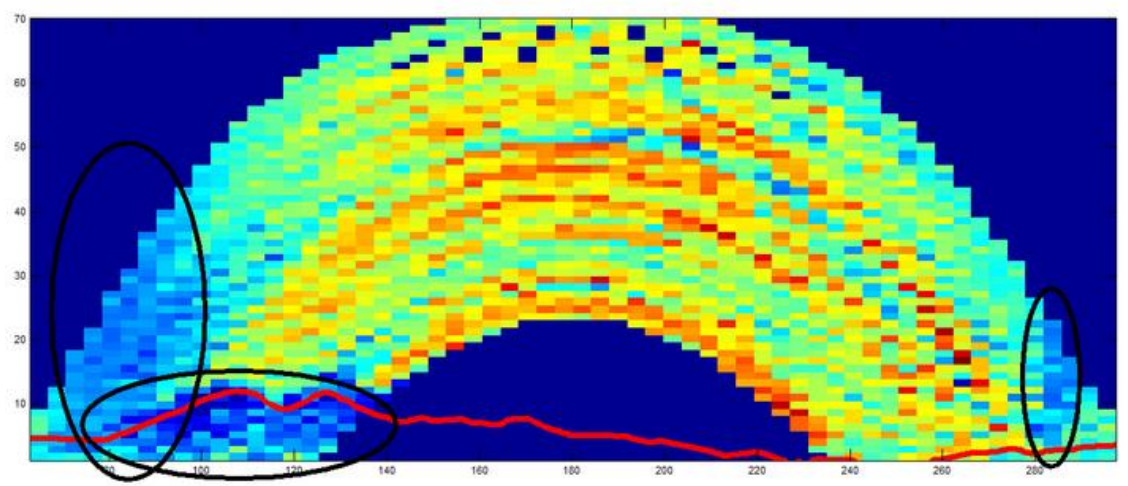

Fig. 4: Azimuth / elevation cumulated view of DNI timeseries. The obstruction by topography (red lines) and presumably trees (black ellipses) can be seen.

He concluded, that automatic and visual quality checks are mandatory and new automatic checks to be determined for tilted pyranometers and spectroradiometers are needed. He proposed to create different levels of processing (as used e.g. for satellite data): Level 0-2 (raw - flagged raw - filtered and complete data).

For the extension period of the Task 16 we propose to issue a thorough QC report of BSRN station in collaboration with BSRN communities.

Gap filling is needed as there is in almost every timeseries missing values or data excluded by QC. For many needs complete datasets are needed - e.g. TMY datasets or aggregated averages.

Manajit Sengupta (NREL, USA) and Adam Jensen (DTU, DEN) showed their procedures of QC procedures. On behalf of Ana Garcia (JRC, ITA/EU) showed Philippe Blanc showed how satellite data can be used to control ground data (Urraca et al, 2017).

\subsection{Workshop on probabilistic forecasting}

This workshop showing the current state of work concerning probabilistic forecasts was organized by Jan 
Remund, Meteotest as a side event of the EUPVSEC in Marseille, France, September 2019. We will show the main results and conclusions. A general introduction was given by Philippe Lauret (Univ. La Reunion, FRA).

\subsection{Workshop on site adaptation methods}

This workshop is organized by Jesus Polo, Ciemat, Spain as a side event of SWC 2019 based on the input of the benchmark exercise (2.1).

\section{Outlook}

In 2020 the newly gained know-how will be concluded in a new update of the solar resource handbook (Sengupta et al., 2017). An extension of the Task will be initiated for the period of 2020-23.

\section{References}

Sengupta, M., Habte, A., Gueymard, C., Wilbert, S., \& Renné, D., 2017. Best Practices Handbook for the Collection and Use of Solar Resource Data for Solar Energy Applications. https://doi.org/10.18777/ieashctask46-2015-0001

Urraca, R., Gracia-Amillo, A. M., Huld, T., Martinez-de-Pison, F. J., Trentmann, J., Lindfors, A. V., ... Sanz-Garcia, A., 2017. Quality control of global solar radiation data with satellite-based products. Solar Energy, 158(August), 49-62. https://doi.org/10.1016/j.solener.2017.09.032 